[[Forthcoming in The Philosophical Quarterly - this is an uncorrected draft, so please don't quote or cite this version!]]

\title{
Perceptual experience and degrees of belief
}

\author{
Thomas Raleigh \& Filippo Vindrola ${ }^{1}$
}

\begin{abstract}
According to the recent Perceptual Confidence view, perceptual experiences possess not only a representational content, but also a degree of confidence in that content. The motivations for this view are partly phenomenological and partly epistemic. We discuss both the phenomenological and epistemic motivations for the view, and the resulting account of the interface between perceptual experiences and degrees of belief. We conclude that, in their present state of development, orthodox accounts of perceptual experience are still to be favoured over the perceptual confidence view.
\end{abstract}

Keywords: Philosophy of mind \& cognitive science; epistemology; perceptual experience; justification; perceptual confidence

\section{Introduction}

What is the relation between our perceptual experiences and our degrees of belief? Jeffrey (1968,

3) famously suggested that this is a merely psychological question. Experiences may causally affect how confident we are in some proposition but there is, according to Jeffrey, no rational relation to unfold here. However, it can seem extremely plausible that there is a rational relation between our experiences and our degrees of belief: our experiences can themselves provide (at least some measure of) rationalization for some specific degree of belief rather than another. They can provide a reason for being more or less confident that something is the case. But so then the question arises: in virtue of what does an experience rationalize one specific degree of belief rather than another? What must perceptual experience be like in order to play this role?

One recent answer to this question comes from the idea that there are perceptual confidences: perceptual experiences not only have a representational content, they also assign degrees of confidence to their content - see e.g. Morrison (2016, 2017); Munton (2016); Moss (2018). The

\footnotetext{
${ }^{1}$ This paper was co-authored as a total collaboration from start to finish, with equal contributions from both authors. The order of the names is merely alphabetical with no further significance.
} 
motivations for this idea are (i) partly phenomenological and (ii) partly epistemological. (i) It is claimed that perceptual confidences provide the best explanation for certain phenomenal features of experiences. (ii) It is claimed that perceptual confidences offer the best explanation for how perceptual experiences can rationalize some specific credence - i.e. a specific degree of belief - in a proposition.

The notion of perceptual confidences is an intriguing and potentially promising idea, and its proponents should be applauded for developing a novel account of perceptual experience. In this paper we explore the relations between perceptual experiences and degrees of belief and outline a number of challenges for the perceptual confidences view, concerning both (i) its phenomenological adequacy and (ii) its account of the epistemic role of experience. ${ }^{2}$

\section{Perceptual confidences: two core ideas}

The idea that perceptual experience embodies a degree of confidence in its content (either in relation to the content or in the content itself) has been given a number of presentations in the literature. For present purposes, it will be useful to identify two core ideas, a descriptive and a normative idea, which we take to be central to the theory and which will be our focus in what follows.

2.1. The descriptive claim is that by appealing to an experience's perceptual confidence, we can account for certain aspects of the phenomenal character of perceptual experiences that it would be hard to explain by appeal only to standard propositional contents. Various examples have been given to support this claim.

Munton appeals to a sequence of changing visual experiences in which a tree gradually goes from being wholly obscured by fog to being wholly and clearly visible (FOG). She also appeals to the continuum of experiences occurring as a subject turns her head so that an object - in this case a "medium-sized grey box" - is initially seen "only out of the corner of her eye", and then "looked at straight" by the subject. Munton describes both of these sequences in terms of the experience of the object in question becoming 'clearer'. She then goes on to claim that the changing phenomenal characters of these experiences are neatly explained by the perceptual confidence view, concluding that "visual confidences provide a powerful tool to describe some of the otherwise hard-to-capture distinctions in the contents of our visual experiences" (Munton 2016, 317).

Morrison provides five more examples in support of perceptual confidences: (a) The visual experience of being in an initially pitch-black room as a light is very gradually turned on, so that it only gradually becomes visible. (b) Looking at the colour of a cloth as the lighting in the room is

\footnotetext{
${ }_{2}$ Other recent papers discussing the Perceptual Confidence view are Beck (2020), Cheng (2018), Nanay (2020), Siegel (forth). We are
} sympathetic to some of the points they make, but due to space constraints we cannot rehearse and discuss their arguments here. 
gradually increased from dark to light. (c) Reading different letters on an optometrist's eye chart, some of which appear too blurry to identify whilst others are clearly recognizable and with many letters falling somewhere in-between. (d) Watching a ball being rolled two different distances in a straight line and then reporting an estimate of those distances. (e) Estimating the number of lines in a drawing, or the precise shape of an oval in a drawing, when the lines/oval are only seen in one's peripheral vision. On the basis of these examples, he goes on to claim that "in each case Perceptual Confidence seems to correctly describe ... how certain objects look to us", and that it "describes these experiences better than [the alternative view] Post-Perceptual Confidence" (Morrison 2016, 16), according to which degrees of confidence are assigned only at the doxastic level. ${ }^{3}$

The core descriptive idea in common here is that all these various instances of what one might call changing levels of phenomenal 'unclarity' are best explained by the alleged changes in perceptual confidence levels of the various experiences.

2.2. The normative claim is that perceptual confidences explain how an experience can in itself provide some measure of rationalization for a specific credence (i.e. degree of belief) in a proposition. This normative part of the perceptual confidence theory can itself be divided into two claims: firstly, that a perceptual experience can in itself, i.e. directly, provide some justification for having a specific doxastic degree of confidence in a proposition. ${ }^{4}$ Secondly, that it is perceptual confidences which explain this normative role. One main motivation for the view then is that it would allow us to endorse not only the idea that perceptual experiences can themselves provide some direct justification for doxastic states but also the idea that specific credences can be thus rationalized. ${ }^{5}$

While Munton and Moss are both perfectly explicit that they take the perceptual confidence view to have important epistemic advantages, Morrison deliberately refrains from talking about justification, preferring to frame the relation between experience and belief in terms of the question "what is it to completely trust your experience?". But despite Morrison’s explicit disavowal of any normative commitments, the notion of "completely trusting one's experience" still comes with a normative component. Trust comes with a 'rationality constraint' (cf. Beck 20206). Compare: if I am fully trusting your testimony that there is a $50 \%$ chance of rain, then (relative to that state of trust) I rationally ought to accept that there is a $50 \%$ chance of rain. If instead, in response to your testimony, I form some other level of confidence that it will rain, then I have responded irrationally -

\footnotetext{
${ }^{3}$ For a discussion of Post-Perceptual confidences see among others Denison (2017), Block (2018), Gross (2018).

${ }_{4}^{4}$ The idea that perceptual experience can in itself provide some direct justification or rationalization for a doxastic state contrasts with the idea that experience can only ever indirectly justify a doxastic state via an inference from some further belief - e.g. a belief about the reliability or accuracy of the experience. Notice that the idea here is just that experience in itself provides some measure of rationalization - not that an experience can all by itself provide outright justification for a belief/credence.

${ }^{5}$ See e.g. Munton (2016, 304): "[there is] a tension between the following four principles, (1) Direct Visual Justification (External World): Some external world beliefs are directly justified by visual experience. (2) Content: a visual experience directly justifies belief in virtue of its contents. (3) Degree: Direct visual justification comes in degrees (4) Binary: Visual states are not degreed. I shall argue that we should abandon (4), accepting instead that visual states present their contents with varying degrees of confidence."

${ }^{6} \mathrm{We}$ are in complete agreement with Beck (2020) here, who writes: "One fairly uncontroversial thing we can say is that when you form a belief as a result of completely trusting your experience, the relation between your experience and your belief is not merely causal. It's also rational. The content of your experience evidentially supports the content of your belief. All things equal, you thus acquire some (defeasible, pro-tanto) warrant for your belief in virtue of having the experience.” (Beck 2020, 11).
} 
given that I am supposed to be fully trusting you. Likewise, were one to completely trust a perceptual confidence as the sole basis for forming an opinion whether $\mathrm{p}$ (whether or not such trust could itself ever be rational), the experience would then rationalize one specific credence in $\mathrm{p}$ rather than any other (relative to that state of trust). The notion of completely and solely trusting one's experience when forming a belief thus plausibly captures the idea that an experience can, in itself, provide some measure of direct rationalization for a specific degree of belief - i.e. without the involvement of any inference from some further belief. ${ }^{7}$

The descriptive and the normative claim are connected insofar as it is supposed to be the same distinctive phenomenal features of experience, which are allegedly well-explained by perceptual confidences, that provide the justification for holding a specific credence in p. It is supposed to be in virtue of a distinctive, less than fully confident way that things look that a perceptual experience can itself rationalize a specific credence. If these two core claims were vindicated, the perceptual confidence theory would seem to provide a neat and relatively simple account of the relations between perceptual experience and degrees of belief. This would be a significant achievement! Not only would perceptual confidence theorists have uncovered a kind of graded structure to the content of experience, they would also be able to provide an answer to an important unsolved question in epistemology.

In what follows, we explore both the phenomenal and the epistemic side of the perceptual confidence proposal, and outline some issues and challenges for the view.

\section{Phenomenological Issues}

In this section, we defend standard representational theories of perceptual experience, and discuss two potential challenges for the descriptive side of the perceptual confidence view. Firstly: appealing to the indeterminacy of representational content seems to offer a powerful rival explanation of the 'unclear' phenomenal features of experience that perceptual confidences are meant to explain. Moreover, such indeterminacy is already a standard commitment of representational theories of perceptual experience. Secondly: the phenomenology of imaginative experience is importantly similar in respect of its 'unclarity' to the features of perceptual experience that are supposedly explained by perceptual confidences. But it is plausible that this distinctive kind of phenomenology in episodes of sensory imagining could not be explained by appeal to graded confidences.

3.1 It is a pretty standard assumption within representational frameworks for experience that spatial properties are represented only up to a certain degree of determinacy. A normal successful perceptual experience accurately represents an object as having a determinable kind of shape, size

\footnotetext{
${ }^{7}$ It worth also mentioning Moss (2018), who argues that positing probabilistic elements in experiences can explain how we can even come to have probabilistic knowledge directly from perception: "it would be especially nice for advocates of probabilistic knowledge if some perceptual experiences did have probabilistic contents, since then an experience could ground your knowledge of its content directly, without the help of any antecedent knowledge" (Moss 2018, 100; emphasis added).
} 
and location, without representing any perfectly determinate spatial property. My experience might represent you as being about $6 \mathrm{ft}$ tall without representing that you have any more determinate height in particular. Likewise, my visual experience represents that a billiard ball has a shape that is more or less smoothly spherical - i.e. up to a certain degree of determinacy - without representing that the ball is perfectly smooth or perfectly spherical. Just to give one example of a representational theorist explicitly stating the determinable nature of perceptual content, consider Peacocke's influential "scenario content" view: ${ }^{8}$

In giving the content we should consider a set of ways of filling out the space ... Greater acuity corresponds to restriction on the set of ways of filling out the space whose instantiation is consistent with the correctness of the representational content. (Peacocke, 1992, 107)

When I see you and your height, there is a whole range of different specific heights around $6 \mathrm{ft}$ tall that you could be consistent with my experience being veridical. When I see the billiard ball, there is a whole range of roughly smooth and spherical shapes that the ball could be consistent with the experience being veridical. Greater acuity of vision, and perhaps also attention, can narrow this range down, as your height, or the ball's shape, is represented with greater determinacy. And this idea is also very naturally extended to colours; when one sees a coloured item only very briefly, or in poor lighting, or in the periphery of one's vision, etc., the visual experience might represent the item as being, say, dark-coloured without representing any more determinate shade of colour.

The fact that contents can represent only a determinable property, without representing anything fully determinate, is an inherent feature of most representational systems. It is familiar that a sentence can represent a determinable colour without representing any more specific shade; it is familiar that a map or diagram can represent various topological features of a spatial arrangement without representing absolute distances. That content can be indeterminate in this way is inherent in the very idea of representing something to be the case. Moreover, it is often claimed that an advantage of representational accounts of experience is that, by appealing to the indeterminacy of representational content, it can explain various kinds of phenomenological indeterminacy - such as blurriness of vision or 'speckled hen'9 cases. And there are robust empirical results showing that shifts in attention can measurably affect the determinacy with which environmental properties are represented. ${ }^{10}$

The idea that perceptual experiences represent properties with more or less determinacy is thus an orthodoxy amongst representational theorists. This is a worry for the Perceptual Confidence theory insofar as representational theorists could alternatively explain the various cases of

\footnotetext{
${ }^{8}$ Cheng (2018) provides an argument for post-perceptual confidences based on Peacocke. But his account relies on Peacocke's notion of 'matching profile', not on the 'scenario content' proposal.

${ }_{9}^{9}$ See Chisholm (1942) - though Chisholm credits the example to Gilbert Ryle.

${ }_{10}$ As an example, consider experiments involving a 'Gabor Patch', seen only in one's peripheral vision, looking more or less blurry and indeterminate depending on the focus of one's cognitive attention. See Gobell and Carrasco (2005) and Yeshurun and Carrasco (2008) for a review of the empirical work; see Stazicker (2011), Nanay (2010), Ganson and Bronner (2013) for philosophical interpretation of these results in terms of determinacy of representational content.
} 
phenomenal unclarity by appeal to the indeterminacy of perceptual content. Given that the possibility of indeterminate content is plausibly intrinsic to the very idea of representation, this would offer a more 'economical' story - we wouldn't have to endorse the additional theoretical machinery of perceptual confidences in order to explain the sorts of cases of phenomenal unclarity that perceptual confidence theorists have pointed to.

For example, consider Munton's FOG case. Changes in the determinacy with which the tree's spatial outline and location (etc.) are represented would offer just as good an explanation for the changing phenomenology of seeing it through gradually dissipating fog. Likewise, consider Morrison's optometrist's example. Here again, the determinacy with which the letter's shape is represented would seem to offer at least as good an explanation, without any appeal to a perceptual confidence. Indeterminacy of content would also seem well-suited for explaining the phenomenology of seeing an object in one's peripheral vision. And if we allow that colour and illumination properties can also be represented with greater or lesser determinacy, then we have a determinacy-based explanation for cases of seeing colour where lighting is poor, or turned on very gradually.

So indeterminacy-based accounts would seem to provide an equally plausible and more economical explanation for the sorts of cases, mentioned in section 2.1 (above), which involve perceiving properties like shape and colour. Here one might object that indeterminate content is not able to explain cases that involve perceiving 'higher-level' properties, which are not part of a hierarchy of more or less determinacy. For example, Morrison suggests that an experience can have a perceptual confidence in a content about a specific individual - 'That is Isaac'. And the property of being Isaac is not part of a hierarchy of more or less determinate properties.

In response, firstly it should be noted that the inclusion of such high-level properties in the content of experience is a highly contested matter, which requires accepting a very "Rich" account of perceptual contents. But secondly, even granting that such high-level properties are indeed part of the content of perceptual experience, changes in the determinacy of the representation of low-level properties could still account for the changes in phenomenology. A content like: "That is Isaac," or: 'That looks like Isaac", could be perceptually represented in virtue of representing various lower-level properties, such as shape and colour - just as the content: 'That is a tetrahedron' could be perceptually represented in virtue of representing the object's triangular faces, or its edges and corners. And so the content 'That is Isaac' might be presented with more or less phenomenal clarity in virtue of changes in the determinacy of the low-level properties that are represented. As Isaac approaches, your experience might come to represent more determinately such properties as his shape, or the colour of his hair (eg first reddish, then red, then scarlet ${ }^{11}$ ), and so the property of 'being Isaac' could be perceptually presented with more phenomenal clarity or force.

\footnotetext{
${ }^{11}$ C.f. Nanay(2020).
} 
Another family of conscious phenomena, which Morrison claims are well explained by the Perceptual Confidence view, are cases in which it can be said that an object looks more like one thing than another. For example, in Morrison's cases of looking at letters on the optician's chart, as well as appearing unclear, a letter might 'look more like an E than an F'. And likewise when looking at the distance a ball has rolled, the distance may look more like it is $10 \mathrm{~m}$ away than $6 \mathrm{~m}$ away. On the perceptual confidence view, the experience assigns a higher confidence to the letter being an E and a lower confidence to its being an F, a higher confidence to the distance being 10 than to its being 6. But one might worry that it is not so clear how appealing to the indeterminate/imprecise nature of representational content can explain this kind of comparative looks property. In response to this latter worry, there seem to be at least 3 ways of responding:

(i) Firstly, as with the 'Rich' properties mentioned above, it is not at all obvious that these kinds of comparative looks properties are something that should figure in the experiential content, rather than being a matter of our downstream cognitive or recognitional reactions to experience. It is at least controversial whether it really is a part of the visual, sensory phenomenology that the letter is more like an $\mathrm{E}$ than an F. And so one option for opponents of the visual confidence view is simply to insist that this kind of comparative appearance is a matter of how we react to and recognise and classify the way that things are visually presented rather than being a part of the visual presentation itself. How (if at all) to draw the perception-cognition divide is itself also a matter of controversy. But one common way to distinguish perceptual experiences from cognitive experiences holds that the mark of cognitive experiences is that they are associated with the tokening of concepts and/or metacognitive assignments/feelings of confidence to their contents (Proust 2013, Weiskoptf 2015, c.f. Beck 2020). Opponents of perceptual confidences might take this to support both the 'post-perceptual' view that assignments of confidence to representational contents fall on the 'cognitive' side and also that these kinds of comparative looks should fall on the 'cognitive' side as well.

(ii) More importantly, this kind of comparative appearance property could in fact be plausibly explained by appeal to indeterminate representational content. Recall here the classic example of the speckled hen ${ }^{12}$, a case which theorists have often sought to explain by appeal to the hen being represented as having an indeterminate number of speckles. It is presumably true when we look at such a hen, which in fact has, say, 42 speckles, that it looks more like it has 40 speckles than 14 speckles, or 400 speckles. The hen is represented as having some number of speckles that is roughly in the range, say, 25-60, though without any such sharp boundaries. We have no natural language concept for this particular indeterminate range of numbers. But assuming for the moment that the experience represents something like this indeterminate content about the number of speckles, this could presumably explain why it is true to say that the hen looks more like it has 40 speckles than it has 14 or has 400: since 40 falls squarely within the indeterminate range (25ish - to - 60ish speckles), whereas

12 See footnote 9 , above. 
neither 14 nor 400 fall within this range. Conversely, the hen presumably does not look more like it has 44 speckles than 40 speckles, since these numbers both equally fall within the range $(25 i s h$-to 60ish). Likewise then with an indeterminate content about the shape of a letter on the optician's chart that is seen with less than optimal clarity. Again, this indeterminate shape-content is not something we have a natural language concept to express. But the indeterminately represented borders and contours could presumably be such that they explain why the letter looks more like an E than an F: for the shape of a letter $\mathrm{E}$ falls somewhere within the indeterminate range of shapes whereas the shape of a letter $\mathrm{F}$ does not. And equally, an indeterminate representation of a distance - which we might crudely express in language with the phrase 'roughly 10 metres away' - could likewise explain why it looks more like 10 metres than like $6 \mathrm{~m}$.

(iii) Finally, some theorists contend that as well as representing physical shapes and colours (etc.), experience can also represent appearance properties 13 - e.g. an experience might simultaneously represent a penny as having the spatial property of being circular and also as having the appearance property of looking elliptical. If we are going to allow that experience can ascribe these kinds of appearance properties, then we don't need to appeal either to perceptual confidences or to indeterminate content. For the experience could simply represent that an object has the appearance property: looks more like an E than an F, and this represented appearance property somehow 'shows up' in the phenomenal character. So if we think that this kind of comparative looks property is part of the genuinely visual, phenomenal character of experience, then one possible explanation is simply that such looks properties can be represented in the phenomenal content of experience. Neither indeterminacy of content nor perceptual confidences would then be needed ${ }^{14}$.

In summary: the idea that perceptual content can represent properties with more or less determinacy offers at least as powerful a tool for capturing the sorts of 'unclear' aspects of experience that were supposed to be motivating the perceptual confidence view. Given that indeterminacy of content is something that almost all representational theorists will be committed to anyway, the need to account for these cases of 'unclear phenomenology' does not provide a motivation to accept the perceptual confidence view. Can appealing to the indeterminacy of content also explain the changes in doxastic confidence that would rationally result in response to such experiences? Well, prima facie, if an object's shape and colour are perceptually represented with more determinacy, this would seem to explain why it is rational to have more doxastic confidence that 'This is a tree', or, 'This is Isaac'.

\footnotetext{
${ }^{13}$ E.g. Shoemaker (1994). For critical discussion see Egan (2006).

14 And after all, having such a comparative appearance property does not seem to have anything especially to do with the kinds of phenomenal unclarity that perceptual confidence theorists have been primarily interested in. For example: when I look at a circle in perfectly clear, optimal viewing conditions, there is no obvious phenomenal unclarity concerning its shape, but this circular shape still presumably has the comparative appearance property of: looking more like an ellipse than a 7-pointed star. And likewise, when I see a new born baby's face in perfectly clear optimal viewing conditions, it looks more like John Lennon than like Paul McCartney. Why should we think then that having these comparative appearances must involve some kind of perceptual uncertainty?
} 
However, we have not yet tried to explain whether and how the determinacy of a perceptual content could rationalize a specific subjective probability distribution. We return to this issue in section 4.3, below ${ }^{15}$.

3.2 Episodes of conscious sensory imagination can exhibit a very similar kind of 'unclarity' to some of the abovementioned cases of unclear perceptual phenomenology. For example, it seems that we can visually imagine an oval shaped object, where the phenomenal character of this imagined oval “doesn't just look to have a particular elongation" (Morrison, 2016, 19), in a way that is at least very similar to seeing an oval in one's peripheral vision. Likewise an imagined object can phenomenally appear to be, say dark-coloured, without appearing to have a particular colour in a way that is, again, at least very similar to seeing a coloured object in one's peripheral vision. Moreover, different sensory imaginative episodes might differ in how clearly or vividly the object's shape or colour appears, just as with different perceptual experiences.

The most extreme version of the idea that perceptual experience and sensory imagination can have similar phenomenal characters would be that they can sometimes have identical or at least indiscriminable phenomenal characters. A number of theorists ${ }^{16}$ have interpreted Perky's classic experiments ${ }^{17}$ as showing that perceptual experiences can be confused for imaginings due to their similar phenomenology. Some have argued that the reverse is also possible - i.e. imaginings can be confused with perceptual experiences. ${ }^{18}$ But note that one does not have to endorse anything this strong in order to accept that both perceptual experience and sensory imagination can at least exhibit importantly similar kinds of phenomenal unclarity in the way that the perceived or imagined object is presented in consciousness.

Let's suppose for the moment that there is a significant similarity between perceptual experience and conscious sensory imagination in respect of their both displaying similar, varying levels of phenomenal unclarity. We might expect then that whatever feature of the content of experience explains the distinctively unclear phenomenology in the perceptual case will also explain the similar unclarity in the imaginative case. However, positing a confidence relation as an intrinsic part of the content of conscious sensory imagination is much harder to make sense of. For imaginative episodes are, at least in general, not committed to (or confident in) their content to any degree whatsoever. If I am asked to imagine a red oval (not some actual, particular red oval, just a red oval), then this conscious state is not committed to the truth of its content. It would be implausible to suggest that when the red oval is imagined with greater or lesser clarity (detail, vividness, etc.), this phenomenal

\footnotetext{
${ }^{15}$ Morrison and Munton briefly discuss and dismiss cases of indeterminacy. Morrison mentions an alleged advantage of perceptual confidences over indeterminacy-based accounts - we will also respond to this line of thought in section 4.3.

${ }^{16}$ See e.g. Ghijsen (2014), Siegel \& Silins (2015).

${ }^{17}$ In these experiments, subjects were asked to fixate on a point on a screen while imagining various objects (like a banana, a leaf, or a book). At the same time, a faint patch of appropriate color, size and shape was projected onto the screen, just above the threshold of visibility. As a result, the majority of subjects reported that they were imagining, rather than experiencing, what they were in fact seeing on the screen. See Perky (1910), Segal (1972), Nigel (2010).

${ }^{18}$ C.f. Teng (2018).
} 
change is to be explained in terms of the imaginative experience becoming more or less confident in the accuracy of its content. What would the standard of accuracy even be here? There is no actual, particular red oval which the imaginative experience is supposed to measure up to.

Now, when we visually imagine that $\mathrm{p}$ it is plausible that we imagine having a visual experience with a content that $\mathrm{p}$. Here, of course, the imaginative state is not committed to the truth of $\mathrm{p}-$ i.e. the imaginative state is not committed to the content of the imagined mental state. And so likewise it might be protested that: were one to imagine having an experience with a specific perceptual confidence that $\mathrm{p}$, that imaginative state need not itself be committed to any confidence level in the truth of p. That is surely correct. But notice that it would be implausible to suggest that the sort of phenomenal unclarity that occurs in episodes of visual imagination is to be explained in terms of the subject imagining having an unclear experience. And likewise it would be wholly implausible to suggest that the varying levels of phenomenal unclarity that occur in different imaginative experiences are to be explained in terms of the subject imagining perceptual experiences with different levels of phenomenal unclarity. For when I imagine a red oval, and the phenomenal character of this imaginative state is such that there is no specific elongation that the oval appears to have and no specific shade of red that the oval appears to have, this is presumably not (at least not typically) because I am imagining having an unclear experience of a red oval. Quite to the contrary, our imaginative episodes can exhibit various kinds phenomenal unclarity despite our best efforts to imagine the objects as vividly and clearly as we can. One imaginative experience that p may be more or less clear/vivid than another imaginative experience that $\mathrm{p}$ despite the subject imagining having a perfectly clear perceptual experience that $\mathrm{p}$ on both occasions So it seems to be a non-starter to explain the phenomenal unclarity of imagination in terms of the subject imagining having an experience with some less-than-total perceptual confidence level.

If appealing to a confidence relation is a non-starter for explaining the 'unclear' phenomenology of imagination, then to the extent that we would like a unified explanation for the similar phenomenology of both perception and sensory imagination, we can legitimately doubt whether a confidence relation is a good explanation for the 'unclear' phenomenology of perceptual experience either.

In summary: both Munton and Morrison endorsed the idea that perceptual confidences allow for a better explanation of certain aspects of perceptual phenomenal character - or 'how things look to us'. In this section we have raised some doubts whether perceptual confidences really do bring any clear phenomenological advantages. Towards the end of his 2016 paper, Morrison strikes a more cautious note, stating that 'Confidentialism' (the thesis that links degrees of perceptual confidence to phenomenal character) is "just a first step", and that "we'd like to know more about the relation between perceptual confidence and perceptual phenomenology" (p. 32). We share Morrison's caution here and, for the reasons we have just discussed, we would like to know more too. 


\section{Epistemological Issues}

Perceptual confidences seem to promise an appealingly neat and simple account of how experiences can themselves rationalize a specific degree of doxastic confidence: by simply having a parallel level of confidence already encoded into the experience - thus allowing for a simple one-to-one mapping from a degreed structure in experience to a degreed structure in belief. In this section, we suggest that matters are not nearly so neat and simple as may first appear. Firstly, we show that embracing perceptual confidences does not yet allow for a precise norm, governing the transition from perceptual experiences to credences, to be formulated. For the perceptual confidence theory does not tell us anything about the role that the subject's prior beliefs and background information also play in determining a new rational credence in evidential propositions. Secondly, we argue that the picture of a simple one-to-one mapping is undermined, since perceptual confidences could be mapped onto two different epistemic properties, rational degrees of belief or degrees of justification, and that these two epistemic properties might come apart. Thirdly, we put pressure on the underlying idea that in order for a specific credence to be directly rationalized there must be any kind of corresponding degreed structure in the experience. The upshot: just as there seem to be no phenomenological reasons to favour the perceptual confidence view over standard representationalism, there seem to be no good epistemological reasons either.

4.1 Munton, Morrison and Moss all repeatedly refer to the following well-known example by Jeffrey (1968), which shows how perceptual observation can deliver the sort of uncertain evidence that strict conditionalization cannot easily accommodate:

In examining a piece of cloth by candlelight one might come to attribute probabilities .6 and .4 to the propositions $\mathrm{G}$ that the cloth is green and $\mathrm{B}$ that it is blue, without there being any proposition $\mathrm{E}$ for which the direct effect of the observation is anything near changing the observer's degree of belief in E to 1. (Jeffrey 1968, 172)

In the example, the visual experience of the cloth can cause a change in the probability assigned to an evidential statement (like G or B), without pushing that probability up to 1 (as entailed by strict conditionalization). Jeffrey proposed a generalization of strict conditionalization to allow agents to update their degrees of belief on a weighted partition capturing this kind of 'uncertain evidence'. In Jeffrey's proposal, experiences can directly affect the agent's subjective probabilities over an evidence partition, changing them to new values (and without necessarily pushing any of them all the way up to 1). Then, Jeffrey's update rule uses these new probabilities, together with the agent's prior probabilities, to determine the rest of her credences.

However, Jeffrey's rule is just silent on how to get such values, i.e. how to go from experience to the probability of the evidence in the first place. It does not tell what the evidence partition is, and it does not tell what the distribution of values over that partition is. This 'incompleteness' of the 
formalism has naturally led to the question of whether we could identify a rule to determine those values. ${ }^{19}$ Besides Jeffrey himself, Carnap, Field and many others ${ }^{20}$ have suggested that this is an "impossible task", because "there is no way to decide how the non-propositional features of some experience, like the clarity of the observation (or the feeling of certainty connected with it or something similar) justifies one particular set of weights rather than another" (Cassell 2019, 16).

Perceptual confidences may, prima facie, seem very well suited to allow these principles to be precisely formulated. The degree of perceptual confidence in $\mathrm{p}$ captures precisely that "feeling of certainty" connected with an experience of $\mathrm{p}$, that could then directly rationalize the very same degree of belief in $\mathrm{p}$. Thus, the evidence partition could be directly determined via the target experience, and the weights over it be rationalized by perceptual confidences. The consequence: a neat and simple account of uncertain evidence, that provides the probability that the visual evidence receives (which can then be plugged into Jeffrey rule).

The alleged epistemic benefits of this new approach would then extend beyond the debate on Jeffrey conditionalization. A large body of literature in (formal) epistemology has been concerned with how to update our credences as we collect evidence - Jeffrey's rule is just the most influential proposal. But how to go from experience to the initial credence in the evidential propositions, regardless of what rule later integrates these evidential beliefs within the rest of your beliefs, remains an open problem. ${ }^{21}$ Perceptual Confidence may look to be a promising candidate to provide an answer.

Proponents of perceptual confidences have advertised this as a highly desirable feature of their proposal, explicitly invoking Jeffrey's original example:

Jeffrey is concerned with the difficulties this [Jeffrey's example above] raises for Bayesian conditionalization, which assumes that the evidence on which we update is accorded a probability of one. Jeffrey conditionalization is a response to this. It allows that the evidence on which a subject updates need not have a probability of one. But this raises a prior question which has so far gone unanswered: what determines the probability that visual evidence receives? How does that evidence take a graded form, if the experiential state itself is binary? (Munton 2016, 310; emphasis added).

The view provides one simple account of what happens when you see the cloth [in Jeffrey's example above], namely that your experience has the content that the cloth is probably green and thereby directly constrains you to have high credence that it is green ... If the content of your experience is that the cloth is .6 likely to be green, you could update your credences by Jeffrey conditionalizing on that content. (Moss 2018, 96)

\footnotetext{
${ }^{19}$ For example, in the form of "a supplementary rule for Jeffrey conditionalization that maps sensory experiences to input distributions, which can then be plugged into Jeffrey Conditionalization" (Weisberg 2009, 10).

${ }^{20}$ Carnap makes this point in a letter to Jeffrey (1975), and Field in his (1978). See also Christensen (1992), Pryor (2013), Miller (2017).

${ }^{21}$ Though see Brössel (2017). For the purpose of this paper, we focus on Jeffrey conditionalization alone, and leave this more general issue for further work.
} 
Perceptual Confidence theorists have all suggested that the rational link from perceptual confidences to credences takes the form of something like an 'expert function'22 - Moss appeals to testimony principles, Morrison to trust relations, Munton to the Principal Principle, explicitly advancing the informal principle that one's credence in $\mathrm{p}$ ought to match one's perceptual confidence that $\mathrm{p}$ - at least in the absence of defeaters:

Both high and low credences can be equally justified, in virtue of their correspondence with the relevant visual confidence. By forming credences that are in line with wellcalibrated visual confidences an individual will in effect be conforming to a version of David Lewis' (1980) Principal Principle, according to which a rational agent conforms their subjective credences to the objective chances. What if the agent fails to conform their credence to the confidence of their experience? In that case we can evaluate the degree of justification that the credence they do form enjoys. The further it departs from the credence that is warranted by the experience, the weaker its justification. (Munton 2016, 322)

In the following, we explore two possible ways to formulate a principle along these lines, telling what credences are then 'warranted' by our experiences. We call it a Perceptual Expert Function (PEF), starting with a very simple version, which we label the Simple Perceptual Expert Function (SPEF):23

(SPEF) If $\mathrm{Cr}$ is your credence function, $\mathrm{E}$ is your previous evidence, and you have an experience with content $\mathrm{p}$ and perceptual confidence $r$, then your new credence in $\mathrm{p}$ should be $\mathrm{r}$.

SPEF states that a perceptual confidence $r$ in $\mathrm{p}$ justifies an agent $\mathrm{S}$ in having a degree of belief $\mathrm{r}$ in $\mathrm{p}$. In Munton's FOG example, a tree-belief-with-degree-r is directly justified by a tree-experience-withconfidence-r; conversely, the most rational response to a tree-experience-with-confidence-r is a treebelief-with-degree-r. Likewise, for the other examples provided in support of perceptual confidences.

But it is fairly obvious that SPEF is too strong to be the correct normative principle. E.g. take standard Muller-Lyer cases: if I am already aware of the illusion - so that E includes the proposition 'the lines in the drawing are equal lengths' - then my experience's high perceptual confidence that: one line is longer than the other, cannot rationalize a high degree of credence in this latter proposition. Or suppose I know I am generally overconfident in certain kinds of perceptual decisions; then I should not always align my credences to my perceptual confidences. ${ }^{24}$ More generally, very often perceptual confidences ought not overrule all the information in $\mathrm{E}$, since $\mathrm{E}$ may contain defeaters for the perceptual evidence. The need to deal with such possible defeaters is explicitly noted by perceptual confidence proponents: "the account presented here [Perceptual Confidence] describes the justification visual states provide for belief in the absence of defeaters or other relevant background features" (Munton 2016, 323, emphasis added). We might then try to restrict PEF so that it

\footnotetext{
${ }^{22}$ The term 'expert function' derives originally from Gaifman (1988), who used the label 'expert probability' for a probability function that a subject is trying emulate with her own probability assignments.

${ }^{23}$ In what follows, we will often talk about single propositions rather than partitions for the sake of exposition, but everything we say is easily translatable to partitions.

${ }^{24}$ Both cases are mentioned by Morrison and Munton.
} 
applies to just those cases where deferring entirely to the 'expert testimony' of our senses seems rationally appropriate. But how would this be precisely formulated?

Consider a weaker version of PEF, that we call the Weakened Perceptual Expert Function (WPEF):

(WPEF) If $\mathrm{Cr}$ is your credence function, $\mathrm{E}$ is your previous evidence, and you have a perceptual experience with content $p$ and perceptual confidence $r$, then your new credence in $p$ should be $r$, provided that your prior evidence $(\mathrm{E})$ is probabilistically independent of $\mathrm{p}$.

WPEF states that, so long as the information in $\mathrm{E}$ has no bearing on $\mathrm{p}$, a perceptual confidence $\mathrm{r}$ in $\mathrm{p}$ rationalizes an agent $S$ in having a degree of belief $r$ in $p$. In the example of the subject who already knows about the Muller-Lyer illusion, her prior evidence, E, contains the proposition 'The lines in drawings are equal length' which is relevant to the proposition 'One of these lines is longer than the other'. But now WPEF, unlike SPEF, does not require the agent to match her credence in this latter proposition to her experience's perceptual confidence in it. WPEF seems to yield the desired result that: rational agents defer to perceptual confidences only if the deference is appropriate - i.e. when no prior information trumps their perceptual evidence.

Mission accomplished? Alas not. For whilst WPEF does not rationally require the subject to defer to her perceptual confidence when background information contains a rebutting defeater, nor does it offer any positive guidance as to what one should do instead. WPEF does not prescribe anything in these cases - it is completely silent about "which probability the visual evidence receives" when the clause about E's irrelevance to $p$ is not satisfied. Whenever a subject has any prior information that probabilistically bears, even a little, on the content of her experience, then there is no longer any guidance for how to rationally respond to her experience. Presumably, for normal adult humans with typical beliefs about the world, we will almost always have some background information that has some probabilistic bearing on the content of our experience. This weaker formulation of PEF thus seems really too weak.

More importantly, even when the clause is satisfied the principle still has counterexamples. One example: if I look at a red sock and my visual experience has a high perceptual confidence, say .9, that it is red, and I know that the lights in the room are red-tinted, the final clause is satisfied. For the proposition 'the lights in the room are red tinted' is probabilistically irrelevant for the proposition 'the sock is red' before I have the experience of the sock. Still, I should not align my doxastic confidence to my perceptual confidence, as WPEF prescribes - that is, I should not adopt a doxastic confidence of .9 that the sock is red - after all, all objects in this scenario will look red. If the lights in the room make all objects look red, the rational response to my experience is to form a flatter distribution over possible colours of the sock (as all non-red socks look red), rather than adopting a doxastic confidence of .9 that it is red.

How might a Perceptual Confidence theorist respond here? Let's now consider three possible responses and see why none of them seem very promising. 
First, it might be possible to provide some other experience-credence norm, that is neither WPEF nor SPEF. But it is very hard to see what such an alternative principle could be. For it is a core component of the theory that the rational credence to adopt in response to an experience is set by means of the alleged match between perceptual and doxastic confidences. This is supposed to account for why, on the basis of an experience, I ought to end up with that precise degree of belief in p. And to do so, it is not enough to show that our credal responses to experiences can increase/decrease (almost everyone agrees with that): to offer a real advantage over rival accounts, perceptual confidences also need to fix the precise amount of confidence that would be rational in response to the experience. That's why perceptual confidence theorists seem bound to require some kind of 'expert function' regulating the transition from experience to degrees of belief.

Second, one might broadly accept WPEF, but try to modify or revise the final 'relevance' clause. To rule out the counterexamples, one might claim that the clause does not capture the relevant notion of 'relevance' here, and propose a new, more restrictive one. There are two major problems with this approach. (i) We have seen that the clause is already too strong - it allows for very few beliefs (if any) to be rationalized by experiences. A stronger notion of 'relevance' would thus render the clause virtually impossible to satisfy - and hence the epistemic benefit of perceptual confidences in effect null. (ii) The example of the red sock would still hold with a little modification. For, regardless of the clause, the (red-tinted) colour of the light in the room can be part of the content of the red sock experience itself. In this case, like in the original one, it's hard to defend the idea that the rational response to my experience should be to form a high credence that it is red.

Third, one might try to devise a richer notion of perceptual evidence so to avoid the problems above. For example, one could let the subject's background beliefs play a role in setting the credence distribution, leading to a more complex evidence partition - one that, for example, already includes information about the reliability of visual system, or correlations with possible perceptual underminers, thus blocking the counterexamples (c.f. Christensen 1992, Weisberg 2015). However, a proposal along these lines would give up the directness of the transition from experiences to subjective probabilities, and hence a main motivation for perceptual confidences. To set the appropriate probability to adopt in response to experiences, whilst still preserving directness, perceptual confidence proponents understand perceptual evidence as a probabilistic distribution over possible simple contents that do not include the target correlation (contents like "x is green .6", "x is violet .4" etc.), and maintain that these confidence assignments are not a function of background information. ${ }^{25}$ Thus rejecting any of these two claims undermines a main motivation for perceptual confidences itself.

${ }^{25}$ Morrison (2016) quickly mentions as a "further line of research" the extent to which background beliefs and hardwired assumptions can influence perceptual confidences (p. 45), but not in epistemic terms. Moreover, his whole argument presupposes that confidence assignments can't rely on any background feature (p. 31-32; see also Beck 2020, p. 7-8). 
The discussion of the third option points to a general challenge for the epistemic ambitions of the perceptual confidence proposal. As seen above, the advertised hope for the theory was that perceptual confidences would be able to supplement the Bayesian framework with an account of the epistemic role of experiences - one that, for example, rationalizes the probabilities assigned to the sort of uncertain evidence that occurs in Jeffrey's example. This would be to provide a precise normative link that would open-up the "black box" transition from experiences to credences. For example, by means of perceptual expertise principles. But perceptual expertise principles seem to do it in the wrong way. For it is very plausible that the rationalization of the evidential weights requires the epistemic import of our experiences to be a function of background information, as the discussion on updating rules and "holism" shows, and that this background information should be taken into account before agents update on the evidence. The reason is that holistic worries take place in the determination of the evidence partition, before Jeffrey's formalism even kicks in (see Cassell 2019). They take place when the initial probability of the visual evidence is assigned, before we integrate it with the rest of our credences. Perceptual expertise principles would not allow background features to take part in this 'rationalization' - subjects would rather entirely defer their (initial) subjective probabilities to perceptual confidences, at least in some cases.

The resulting picture is a rejection of holism: to save the direct transition from experience to initial credence, hence PEFs, one rejects the idea that background features play a role in determining the initial probability that $\mathrm{x}$ is $\mathrm{p}$. But not many epistemologists would just give up holism so easily. For those who don't, perceptual confidences alone are not enough to directly determine the probability of experiential evidence onto which subject can later update their credences, insofar as the latter should plausibly be a function of background information. If the probability that the evidence receives is often determined by your background beliefs, and confidence assignments are not, it is hard to see how perceptual confidences can determine the target probability. And it is hard to see how they can provide experiential contents onto which we can simply Jeffrey-update on.

Now, to be clear, how to formulate a precise norm governing the transition from experiences to credences, especially one that deals with holism, is a tricky problem that all candidate theories face. So the failure of the perceptual confidence view to provide such a norm is not in itself a decisive reason to reject the view. The point of this section has rather been that the perceptual confidence theory does not yet provide the sort of epistemological benefits that its proponents seem to advertise it as providing - viz, a precise norm for the sort of transition from an experience to a new credence function that occurs in Jeffrey's famous example of seeing coloured cloth by candlelight (to which Morrison, Munton and Moss all refer). So it remains doubtful whether the proposed probabilistic treatment of experience brings any clear epistemic advantage over existing, rival accounts.

At this point a friend of perceptual confidences might reply that: sure, perceptual confidences are not the full story in determining evidential probabilities - some further rule or theory is needed that integrates them with background information. But the perceptual confidence view at least provides a 
neat theory of how experience can make some kind of rational contribution to determining credences insofar as it treats experiences as having just the same kind of graded, probabilistic structure as credences. It is true that the perceptual confidence view has the merit of at least trying to open up the "black box" transition from experience to credence. And we certainly do not want to rule out that the perceptual confidence view could be somehow supplemented so as to yield a satisfying overall picture. However, firstly, we so far have no clue what such a supplementary rule or theory might look like. Secondly, as we will argue in the next two sections, there are reasons to doubt that the apparently simple picture of a graded, probabilistic structure in experience that neatly maps onto the graded, probabilistic structure of our credences is attractive even as a partial and incomplete story of the epistemic relation between experiences and credences.

4.2 It is surely very plausible that when you experience something more or less clearly (in some sense of experiential clarity), this change in clarity can affect the 'epistemic force' of the experience. But there are various ways in which epistemic force could be understood here. Phenomenal shifts in the quality of experiences might modulate the amount of justification for holding some doxastic attitude towards $\mathrm{p}$ that the experience provides. Or they might modulate the level of confidence in $\mathrm{p}$ that is rationally permitted (or most rational) on the basis of the experience. Or perhaps they determine both the amount of justification provided by the experience and the amount of doxastic confidence that is most rational in light of the experience.

But once we have these two different epistemic properties in play, we must consider the possibility that they could come apart - i.e. that epistemically relevant changes in the phenomenology of perceptual experiences might sometimes correspond to changes in just one of epistemic properties, and some other times to changes in the other epistemic property. This would threaten the neat and simple picture of a one-to-one mapping from a single degreed feature in the phenomenal content of experience to a single degreed epistemic feature of belief that motivates perceptual confidence.

Munton takes the basic epistemic explanandum to be that "changes in phenomenal appearance have epistemic upshots: your epistemic position also changes very gradually, in line with them" (p. 3). She initially discusses this change in epistemic position in terms of "degrees of justification", but then comments: "We could ask the same question not in terms of degree of justification but in terms of degree of belief" (p. 303). ${ }^{26}$ One might thus wonder whether perceptual confidences are meant to offer both an account of the degree of justification that full beliefs and credences would (directly) receive from an experience, and the degree of belief (credence) that the subject is rationally entitled to have on the (direct) basis of the experience. On Munton's story, a perceptual confidence that p might look to determine both the amount of justification that the experience confers on doxastic states and to determine which specific credence is most rational in light of the experience.

26 This second reading seems to be also suggested by the quote about the principal principle above. 
However, a change in one's evidence that increases (or decreases) the justification for a doxastic state will not always go together with a change in the degree of doxastic confidence that is most rational in light of that evidence (or vice-versa). This should be immediately clear as soon as we consider that specific degrees of belief can be more or less strongly justified. Some evidence may more weakly justify (to degree n) a credence of, say, 0.75 that $\mathrm{p}$, whilst other evidence may more strongly justify (to degree $\mathrm{m}$ ) that very same $\operatorname{Cr}(\mathrm{p})=0.75$. For example, if my only evidence about the weather is one piece of testimony that the chance of rain is 0.75 from some reliable, expert forecaster, then the credence that it will rain that is most rational for me given my evidence might well be 0.75 , but this credence will be only relatively weakly justified. In contrast, suppose my evidence about the weather includes many forecasts by a whole range of reliable and expert forecasters who all claim that the chance of rain is 0.75 . Once more, my most rational credence that it will rain might well be 0.75 , but this credence will now be much more strongly justified. Conversely, one set of evidence may strongly justify (to degree $n$ ) $\operatorname{Cr}(p)=0.7$, whilst another set of evidence may provide an equally strong degree of justification (to degree $\mathrm{n}$ again) but for a different credence in $\mathrm{p}$ of, say, $\operatorname{Gr}(\mathrm{p})=0.8$. For example, if my only evidence about the weather is testimony that the chance of rain is 0.7 , then that may justify to degree $n$ my $\operatorname{Cr}(\mathrm{p})=0.7$. If instead my only evidence about the weather was testimony from the very same person that the chance of rain is 0.8 , then that may justify to the very same degree $\mathrm{n}$ my having $\operatorname{Cr}(\mathrm{p})=0.8$.

So it is certainly at least possible for the degree of justification that is provided and the degree of confidence that is best-rationalized to vary independently as one's evidence changes. The two epistemic properties need not always covary. We ought not assume then that changes in phenomenal character of experiences that increase (or decrease) the amount of justification provided will also always determine a parallel increase (or decrease) in the rational level of doxastic confidence - nor vice-versa. On the other hand, the fact that there can be some possible evidential changes which allow justification and rational confidence to vary independently should not lead us to assume that there are some possible experiential changes which likewise allow for such independent variation.

So then: is there a clear example of a change in perceptual experience that modulates the justification for a single credence that remains the most rational credence throughout? Well, suppose E1 is a visual experience in which the grey box changes very rapidly from being only very unclearly visible at the edge of one's visual field to being much more clearly visible in the centre of one's visual field - over the course of less than a second. Whereas E2 is a visual experience in which the grey box undergoes just the same transition from being unclearly and peripherally seen to being clearly and focally seen, but this time the change unfolds much more slowly - over the course of 10 seconds. You might think that with both E1 and E2, the degree of doxastic confidence that there is a box which is best-rationalized increases in just the same way from some low credence to a higher credence. But you might also think that in E1 the amount of justification for this specific range of changing credences is relatively weak, due to the rapidity of the phenomenal changes over the experience's 
short duration. Whereas in E2 this very same progression of changing credences receives a higher amount of justification (since the phenomenal changes unfold more slowly).

Here is another example that does not rely on the duration of the experiences in question: suppose that there can multimodal experiences that have some kind of unified phenomenal character, drawing on more than one sensory modality - e.g. both touch and vision. ${ }^{27}$ Now we might have a multimodal experience MM1 which is comprised of both seeing and feeling the shape of some object, where the visual component would in itself best-rationalize $\operatorname{Cr}($ square $)=0.7$ and the tactile component would best-rationalize $\operatorname{Cr}($ square $)=0.9$. Thus overall MM1 best-rationalizes $\operatorname{Cr}($ square $)$ $=0.8$. In contrast, MM2 is also comprised of both seeing and feeling the shape of the object, but here the visual component would best-rationalize $\operatorname{Cr}($ square $)=0.8$ and the tactile component would also best-rationalize $\operatorname{Cr}($ square $)=0.8$. Thus overall MM2 again best-rationalizes $\operatorname{Cr}($ Square $)=0.8$. However, it seems reasonable that MM2 would provide greater justification for this $\mathrm{Cr}$ (Square $)=0.8$ due to the unanimity of the different sensory components. ${ }^{28}$

If there really are pairs of experience that keep the most rational credence constant but provide different degrees of justification for that very same credence, then the alleged perceptual confidences of those experiences could not provide a unified account for both justificatory force and rational credence. More generally, if there are two different graded epistemic properties that can be differentially affected by perceptual experience, then the very idea that we should posit a single graded quantity in the content of experience to explain all its epistemic effects would seem to require a stronger motivation. ${ }^{29} \mathrm{At}$ the least, we would no longer have a nice and simple one-to-one mapping between a graded feature in experience and a graded epistemic feature on the doxastic side.

One might claim that perceptual confidences only explain the varying credences that would be most rational in response to experience and do not explain the varying degrees of justificatory force that experiences can possess. Or vice-versa. But then: firstly, the other epistemic property would have to be explained in some other way, and perceptual confidences would lack an account for that. Secondly, one might wonder how a subject can tell when some change in the phenomenal character of her experience is due to a change in perceptual confidence - thus rationalizing a change in credence - and when the change in phenomenal character is due to some other factor which is instead affecting the amount of justification that the experience would provide. Again, the initially neat and simple looking story comes to seem much less neat and simple on closer inspection.

\footnotetext{
${ }^{27}$ Munton (2016, 302) claims she sees no reason not to extend her account to other sensory modalities, and both Morrison (2016, 11) and Moss $(2018,100-101)$ briefly discuss multisensory integration, though not in epistemic terms, as we instead do.

${ }^{28}$ Now, of course one might want to reject the intuitions that degree of justification and best-rationalized degree of confidence would come apart in these cases. We can also imagine objections to the reliance of these examples on the duration of the experience, on considerations about higher-order evidence or beliefs, or on the idea of a unified multi-modal experience. So we do not want to suggest that these examples are entirely decisive - they are only meant to be suggestive of possible ways that the two epistemic properties might come apart. And whether one finds these examples fully convincing or not, still we would insist that the intuition that credences warranted by experiences and amount of justification can come apart seems hard to resist.

${ }^{29}$ Munton mentions similar problems (p. 319). Still, her discussion does not allow for most rational level of credence and amount of justification of this credence to vary independently. For example, her picture does not allow for a change in experience to increase the justification for the same most-rational level of credence. So she does not deal with the issue we raise here.
} 
4.3 One might think that the perceptual confidence view is still at an epistemological advantage because, unlike orthodox accounts of perceptual content, it at least ascribes to experience the right sort of 'degreed' structure that would allow it to have some kind of direct epistemic bearing on our degrees of belief. An alleged epistemological motivation for the view is that it avoids a tension in trying to have non-graded perceptual contents interface directly with graded doxastic states (whether or not these grades are probabilities). Munton explicitly argues that this tension, or "mismatch, is a problem for rival, orthodox theories of perceptual content", ${ }^{30}$ and is one of the main reasons why we should accept perceptual confidences.

These remarks suggest a commitment to something like the following principle, which we label Graded-Out Graded-In (GOGI):

(GOGI) Only evidence that already has some graded structure could directly rationalize a specific degree of belief (or directly provide a specific degree of justification for belief).

Why accept this principle? The thought is presumably something like this: if the evidence consists of just a plain, old, non-degreed content that p, then there would not seem to be enough 'moving parts' in the evidence to directly determine a specific credence (less than 1) as the most rational to adopt, based solely on that evidence - arriving at a specific credence could only come via some kind of further inference from a belief, e.g. about the reliability of the evidence. Thus the perceptual confidence theorist posits an extra 'moving part', with as many degrees of freedom as the credences they are supposed to rationalize, as an intrinsic feature of the experiential evidence - the alleged confidence relation. Evidence which lacked this intrinsic structure would require some further mediating belief on the subject's part, in order to arrive at a specific level of confidence. (And likewise to arrive at a belief with a specific degree of justification.).

Now, even if GOGI is accepted, one might still resist the perceptual confidence theory by appealing to some other intrinsically graded feature or aspect of the experience - i.e. to some other kind of 'moving part' - that might then map onto specific credences. Even granting that there must be some kind of graded quantity baked into the experiences, there are various possible graded properties other than confidences that the phenomenal character could be somehow encoding in cases such as the FOG experience - like vividness, presentational/assertoric force, or expected precision. ${ }^{31}$ Of course, perceptual confidence proponents might claim that they needn't choose between these candidate gradable properties - all they want to establish is that there is some kind of degree and whether you want to call this confidence or vividness (etc.) is not important. But it is not at all obvious that these different possible graded features in experience are all epistemically equivalent - i.e. it

\footnotetext{
${ }^{30}$ Munton and Morrison both discuss various proposals along these lines.

${ }^{31}$ For a classical example of vividness-based accounts, think of Hume's claim that perception and imagination are distinguished by different degrees of vividness; for a more recent one, consider the claim by many recent theorists that perceptual experience has a distinctively 'presentational' character. For an example of assertoric force, think of how a claim may be asserted more strenuously depending on all sorts of factors, not just one's confidence in its truth - e.g. its importance, urgency etc. For an example of precisionbased accounts see Denison (2017).
} 
is not obvious which of these possible graded features (if any) line up neatly with either rational credence or strength of justification. So even if GOGI is accepted, the perceptual confidence theorist would still owe us an explanation for why we should prefer grade-able levels of confidence, rather than some other grade-able property, as an intrinsic feature of experience.

But a different option would be to reject GOGI altogether, resisting the idea that there must be something in the experience with as many degrees of freedom as the subject's credences in order for the experience to directly rationalize one specific credence rather than another. One might insist that a specific credence function can be a rational response to an experience, but that this response need not be based on any kind of degreed structure in the experience, nor on any kind of inference or further belief about the reliability or evidential bearing of the experience. Let's first consider the plausibility of directly forming a flat probability distribution in response to a simple, non-degreed evidential proposition. Suppose that your evidence includes the proposition that a coin-toss has landed either heads or tails, but that you have no further information whatever about the coin or the toss. Your immediate reaction might well be to divide your confidence evenly between the coin having landed heads and having landed tails. This would, of course, be in line with the famous 'Principle of Indifference'. But it is quite implausible that a belief in this principle needed to function as your reason for forming these credences in order for those credences to be rational, or that any kind of further inference or belief need be involved ${ }^{32}$. Your complete uncertainty as to heads or tails might just immediately be reflected in your having 50:50 credences. And indeed this would be the rational reaction to having this as your only evidence about the coin toss. But clearly the propositional content of the evidence: that the coin landed either heads or tails, has no kind of confidence relation or probability 'baked into' it. So it is at least highly plausible that you could be fully trusting and relying on this non-graded evidence as your only guide to forming your credence, and directly arriving at a specific credence function in rational response.

Let's now consider a different kind of evidential proposition: e.g. 'The stick is about $1 \mathrm{~m}$ long', or 'the shirt is red'. These propositions have indeterminate contents. They represent the object as having a property within some range of properties, but neither the precise location within the range nor the exact limits of the range are specified. So the precise length of the stick is not represented, nor are the precise limits of the range 'about $1 \mathrm{~m}$ '. Likewise, the precise shade of the shirt is not represented, nor are the precise boundaries of red-ness. Whereas above, in the case of the evidence about the coin landing heads or tails, the suggestion was that one might directly and non-inferentially form a flat distribution of credences across the possible options, in these sorts of cases we suggest that a subject might directly and non-inferentially form a bell-curve shaped distribution. If I am fully and solely trusting the evidence that: 'the stick is around $1 \mathrm{~m}$ long', I might naturally distribute my credences concerning

${ }^{32}$ As well as the implausible over-intellectualization of requiring a belief in the principle of indifference, requiring that the subject employ this principle as a further, explicit premise in an inference would seem to open up the sort of regress that Carroll (1895) famously identified in his dialogue between the Tortoise and Achilles. 
the exact length of the stick in a bell-curved shape with its peak at exactly $1 \mathrm{~m}$, tailing away in either direction. This need not, we suggest, be based on any kind of inference or further belief. It could simply be the rational credential response to the indeterminate or imprecise nature of the evidence. Likewise, in response to the evidential proposition: 'The shirt is red', it could be entirely natural and rational to form a credence function about the colour of the shirt which is highest around the prototypical red, and which tails away towards less prototypical red shades. I.e. the rational response to this evidence would not be to form a plateau distribution over possible reds, but rather to privilege some regions of the colour space - those that correspond to more prototypical reds. Again, these indeterminate evidential propositions do not involve any kind of probability or graded confidence structure. Our suggestion then is that if a perceptual experience represents only a determinable shade of red, an immediate and rational response to that way of looking might be a non-flat credence distribution that favours more prototypical areas of red colour-space over less prototypical areas. Or if my experience represents two objects as being about the same size, I might immediately and rationally respond to their visual appearance with a bell-curve shaped credence distribution over possible differences in their size, whose peak is at 0 difference.

Now at this point it might reasonably be objected that such a non-flat, bell-curve shaped probability distribution would not be directly formed as the rational response to indeterminate evidential contents such as: 'The stick is about $1 \mathrm{~m}$ long' or 'The shirt is red'. Rather, the rationality of forming such non-flat distributions would depend on possessing further background beliefs about normal conceptual/linguistic usage - e.g. that the concept 'red' is more likely to be used to refer to prototypical shades of red than non-prototypical shades. In which case it would not be the indeterminate evidential propositions alone that are directly rationalizing the non-flat distribution of credences. And so these cases would not be counter-examples to GOGI after all. However, whilst it is plausible that sometimes, perhaps often, depending on the nature of the evidence, a subject would in fact draw on background information when forming a non-flat distribution in response to such indeterminate evidential propositions, some kind of further argument is required to establish that this must always be the case. Especially in view of how highly plausible it was that one could rationally form a flat probability distribution in direct response to certain kinds of non-degreed evidence (e.g. 'The coin landed either heads or tails'), it would require some further persuasive, positive reason before we rule out the possibility that one might also sometimes rationally form a non-flat probability distribution in direct response to other kinds of non-degreed evidence (e.g. 'The shirt is red') simply by applying the concept RED. It is certainly not just obvious why this should not be possible.

In support of the idea that this is possible, here is one last example of non-degreed, nonprobabilistic evidence for which it seems plausible that one might rationally form a non-flat probability distribution in direct response - i.e. not drawing on any background beliefs about normal conceptual usage, etc. Consider a photograph of a table that is out of focus, so that the edges of the table look like a bit of a blur. One's immediate rational response here, we suggest, might well be a 
roughly bell-curved distribution of confidences about the exact location of the edge, with the highest credence assigned to locations around the middle of the blur. Must this credential response to the blurry image be inferential? Must your credence be based on further beliefs about how blurriness works, or the reliability of the photo? Plausibly not. You might just directly recognize that the blur allows for only a spread of confidence about where the exact location of the table-edge is. And you might naturally and non-inferentially form something like a bell-curve shaped distribution just based on the look of the blurry photo. (And perhaps, if the blurriness of the image had a different kind of look, one might rationally form some other shaped distribution.) The point here is just that it seems entirely possible that a non-flat distribution could be the rational distribution of confidences to have, based solely on a blurry photo as your only evidence. But of course a blurry photograph does not itself embody or encode any kind of less-than-total confidence level in what it represents! It is just an imperfect and imprecise kind of representation. Likewise then, it seems an open possibility that certain kinds of unclear or indistinct perceptual phenomenology could rationally call for a bell-curve shaped credence distribution in response, without the experience itself encoding anything like a perceptual confidence. The experience might just have an indeterminate or imprecise content.

Morrison does briefly consider the idea that we might form a specific credence function in response to indeterminate content:

Once again, post-perceptual confidence can't offer as simple an explanation. Suppose your experience represents a range of elongations [of an oval]. Say: from $\mathrm{O}$ to 0 . By trusting your experience you would end up with doxastic confidences that are plateaushaped: equal confidence in elongations within the relevant range, and no confidence in elongations outside that range. You wouldn't end up with a bell-shaped distribution of confidence. (33)

But as we have seen, a content that ' $\mathrm{X}$ is tall' or ' $\mathrm{Y}$ is red' does not specify a precise and determinate range, with sharp boundaries, that the item falls into. And we have suggested that a natural response to certain kinds of phenomenal indeterminacy could be to form a non-flat, bell-shaped credence distribution. Nothing Morrison says here undermines the plausibility of that suggestion. Thus, it remains at least an open possibility that one could directly and rationally respond to some evidence with a specific degree of confidence, even though the evidence itself does not have any kind of degree or confidence level baked into it. This would be to reject the GOGI principle that the only way experience could 'directly interface' with a specific degree of belief is if the experience itself also has an intrinsically graded structure. It is not obvious that there has to be any feature or 'moving part' in the experience itself (whether or not it is anything like a confidence or probability) that has the same degrees of freedom as our credences, in order for the experience to directly rationalize one specific credence rather than another. 
Now, to be clear, we have not tried to offer here a complete theory of how a specific indeterminate content can rationalize a specific credence function. We have only sought to establish the coherence and prima facie plausibility of rationally forming a specific credence in direct response to evidence, such as a perceptual experience, which lacks anything like a graded confidence level or probability distribution in its content. In other words, we have tried to show that GOGI is far from obvious and might plausibly be rejected, thereby undermining a basic epistemological motivation for perceptual confidences. For if GOGI is rejected, there's no 'mismatch' between standard representational contents of perception and our degrees of belief for the perceptual confidence theorist to resolve. Moreover - as we have tried to show in sections 4.1 and 4.2 , above - the perceptual confidence view does not yet offer anything like a precise theory of how to form specific credences on the basis of experience either. Overall then, we have reason to think that positing the extra theoretical machinery of perceptual confidences will complicate rather than simplify our epistemological picture without yet bringing clear epistemic advantages.

\section{Conclusion}

Although none of the issues we have raised here individually amounts to any kind of refutation of perceptual confidences, we suggest that they cumulatively cast doubt on the theory. But we also hope that our discussion may indicate some possible future lines of research - both for perceptual confidence theories and for more orthodox representational views. In its present state of development, the perceptual confidence view does not yet offer advantages over rival theories in accounting either for perceptual phenomenology or for the normative relations between perceptual experiences and degrees of belief. Of course, the idea of perceptual confidences is very recent and still in its infancy, and we certainly do not rule out the possibility that it could be developed in such a way as to overcome these problems. We would be delighted if the present paper contributed to such future developments ${ }^{33}$.

\footnotetext{
${ }_{33}$ Earlier versions of this paper were presented at the Ruhr-University Bochum, at the Institute of Philosophy in London, at the EEN 2018 conference in Amsterdam, at AISC 2019 conference in Lucca and at the NYU-Abu Dhabi \& UAEU joint conference on Philosophy \& Cognitive Science. We are very grateful to the audiences on all these occasions for their questions and comments. Both authors wish to give special thanks to Peter Brössel, not only for all of the conversations on the topic of this paper, but also for being a superb project leader and supporting us both in so many ways. Special thanks are also due to John Morrison who presented a wonderfully helpful commentary on our paper at the conference in Bochum. Many thanks as well to: Edouard Machery, Insa Lawler, Jim Pryor, Susanna Siegel, Harmen Ghijsen, Bence Nanay, Alfredo Vernazzani, Moritz Schulz, Jake Quilty-Dunn, Carlotta Pavese, Catrin Campbell-Moore, Florian Steinberger, Jennifer Carr, Jona Vance, Ophelia Deroy, Jonathan Najenson. Finally, we are immensely grateful to two anonymous referees for this journal whose insightful and constructive comments greatly improved this paper. Research on this paper was supported by a UAEU Research Start-up Grant (G00003259). We'd also like to thank the German Research Foundation (DFG) which supported this research in the context of funding the Emmy Noether Grant "From Perception to Belief and Back Again" (BR 5210/1-1) and the Research Training Group "Situated Cognition" (GRK 2185/1)/Gefördert durch die Deutsche Forschungsgemeinschaft (DFG) - Projektnummer GRK-2185/1 (DFG-Graduiertenkolleg Situated Cognition).
} 


\section{References}

Beck, J. (2020) 'On Perceptual Confidence and "Completely Trusting Your Experience", Analytic Philosophy.

Block, N. (2018) 'If perception is probabilistic, why does it not seem probabilistic?', Phil. Trans. R. Soc. B: 373 .

Brössel, P. (2017) 'Rational Relations Between Perception and Belief', RPP, 8/4: 721-741.

Carroll, L. (1895) 'What the Tortoise said to Achilles', Mind, 104/416: 691-693.

Cassell, L. (2019) 'Higher-Order Beliefs and the Undermining Problem for Bayesianism', Acta Analytica, 34: 197-213.

Cheng, T. (2018) 'Post-perceptual confidence and supervaluative matching profile', Inquiry, 1-29.

Chisholm, R. (1942) 'The Problem of the Speckled Hen', Mind, 51:368-373.

Christensen, D. (1992) 'Confirmational holism and bayesian epistemology', Phil. Sci., 59/4: 540-557.

Denison, R. (2017) 'Precision, Not Confidence, Describes the Uncertainty of Perceptual Experience comment on John Morrison's “Perceptual Confidence.”, Analytic Philosophy, 58:58-70.

Egan, A. (2006) 'Appearance Properties?', Noûs, 40/3:495-521

Field, H. (1978) 'A note on Jeffrey conditionalization', Phil. Sci., 45:361-367.

Gaifman, H. (1988) 'A Theory of Higher Order Probabilities'. In: Skyrms B., Harper W.L. (eds) Causation, Chance and Credence, 41, Springer, Dordrecht.

Ganson T., Bronner B. (2013) 'Visual prominence and representationalism', Phil.Stud, 164(2): 405418.

Ghijsen, H. (2014) 'Phenomenalist Dogmatist Experientialism and the Distinctiveness Problem', Synthese, 191(7).

Gobell J, Carrasco M. (2005) 'Attention alters the appearance of spatial frequency and gap size', Psych. Sci,16.

Gross, S. (2018) 'Perceptual consciousness and cognitive access from the perspective of capacityunlimited working memory', Phil. Trans. R. Soc. B, 373.

Jeffrey, R. (1968) 'Probable Knowledge'. In Probability and the Art of Judgment, GUP, pp. 30-43.

Jeffrey, R. (1975) 'Carnap's empiricism'. In G. Maxwell \& R. M. Anderson Jr. (Eds.) Minnesota studies in the philosophy of science. Vol. 6, pp. 37-49, Minneapolis: UMinnesota Press.

Lewis, D. (1980) 'A subjectivist's guide to objective chance'. In Ifs, Springer, pp. 267-297.

Teng, L. (2018) 'Is Phenomenal Force Sufficient for Immediate Perceptual Justification?', Synthese, 195/2:637-656.

Miller, B. (2017) 'Updating, undermining, and perceptual learning', Phil. Studies, 174: 2187-2209.

Morrison, J. (2017) 'Perceptual Confidence and Categorisation', Analytic Philosophy, 58/1: 71-85.

Morrison, J. (2016) 'Perceptual Confidence', Analytic Philosophy, 57/1: 15-48.

Moss, S. (2018) Probabilistic Knowledge, OUP.

Munton J. (2016) 'Visual Confidences and Direct Perceptual Justification', Phil. Topics, 44/2:301-326.

Nanay, B. (2010) Perceiving the World. New York: OUP.

Nanay, B (2020) 'Perceiving indeterminately', Thought, 1- 7. 
Nigel, T. (2010) 'Mental Imagery', Stanford Encyclopedia of Philosophy.

Peacocke, C. (1992) 'Scenarios, Concepts, and Perception', in Tim Crane (ed.), The Contents of Experience, CUP.

Perky, C. (1910) ‘An Experimental Study of Imagination', American Fournal of Psychology, 21.

Proust, J. (2013). The Philosophy of Metacognition: Mental Agency and Self-Awareness. New York: OUP.

Pryor, J. (2013) 'Problems for Credulism'. In Tucker (ed) Seemings and fustification (pp. 89-131). OUP.

Segal, S.J. (1972) 'Assimilation of a stimulus in the construction of an image: the Perky effect revisited'. In Sheehan (ed) The Function \& Nature of Imagery (pp. 203-30). NY: Academic Press.

Shoemaker, S. (1994) 'Phenomenal Character', Noûs 28/1:21-38.

Siegel, S. (forth) 'How can perceptual experiences explain uncertainty?', Mind \& Language.

Siegel, S., Silins, N. (2015) 'The Epistemology of Perception', in M. Matthen (eds) Oxford Handbook of the Philosophy of Perception.

Stazicker, J. (2011) 'Attention, visual consciousness and indeterminacy', Mind \& Language, 26(2).

Thomas, N. (2014) 'Mental Imagery', The Stanford Encyclopedia of Philosophy.

Weisberg, J. (2009) 'Commutativity or Holism? A dilemma for Conditionalizers', BfPS, 60/4:793812.

Weisberg, J. (2015) 'Updating, undermining, and independence', BfPS, 66/1: 121-159.

Weiskopf, D. (2015) 'Observational concepts'. In E. Margolis \& S. Laurence (eds.), The Conceptual Mind, Cambridge, MA: MIT Press, 223-248.

Yeshurun, Y., \& Carrasco, M. (2008) 'The effects of transient attention on spatial resolution and the size of the attentional cue', Perception \& Psychophysics, 70/1:104-113.

\section{Affiliations:}

Thomas Raleigh: United Arab Emirates University, United Arab Emirates

Filippo Vindrola: Ruhr-University Bochum, Germany 